\title{
COMPLEX INVERSION THEOREMS FOR GENERALIZED STIELTJES TRANSFORMS
}

ANGELINA BYRNE and E. R. LOVE

(Received 27 November 1972)

Communicated by B. Mond

\section{Introduction}

In this paper we seek to establish some "complex inversion formulae" for the generalized Stieltjes transform

$$
f(s)=\int_{0}^{\rightarrow \infty} \frac{d \alpha(t)}{(s+t)^{p}} \quad \text { for all } s \text { in the cut plane, }
$$

supposing that $p$ is any complex number except zero and the negative integers. The "cut plane" means all complex numbers except those which are negative real or zero.

Widder $[1, \mathrm{ch}$. VIII $]$ gave the classical account of the case $p=1$, his complex inversion theorem [1, page 339, Theorem $7 \mathrm{a}]$ being

THEOREM A. If $p=1, \alpha$ is of bounded variation locally in $[0, \infty)$, and (1.1) converges for some s in the cut plane, then for each positive $x$

$$
\begin{aligned}
\frac{1}{2}\{\alpha(x+0)+\alpha(x-0)\} & -\frac{1}{2}\{\alpha(+0)+\alpha(0)\} \\
& =\lim _{\eta \rightarrow+0} \frac{1}{2 \pi i} \int_{-x}^{0}\{f(s-i \eta)-f(s+i \eta)\} d s .
\end{aligned}
$$

The function $f$ in (1.2) is sufficiently defined by (1.1), because convergence of the "improper" Stieltjes integral (1.1) for one single $s$ in the cut plane implies its convergence for all; this follows from Widder's Theorem 2a [1, page 326]. A similar remark applies to Theorems $\mathrm{B}$ and $\mathrm{C}$, even though $p \neq 1$; it rests on Theorem 1 in $\$ 4$ below.

Sumner [2] gave a complex inversion formula for (1.1) for all real $p>0$; his inversion theorem [2, page 181, Theorem $4 \mathrm{~b}$ ] being

THEOREM B. If $p>0, \alpha$ is of bounded variation locally in $[0, \infty)$, and (1.1) converges for some $s$ in the cut plane, then for each positive $x$ 


$$
\begin{aligned}
\frac{1}{2}\{(\alpha(x+0)+\alpha(x-0)\} & -\frac{1}{2}\{\alpha(+0)+\alpha(0)\} \\
& =\lim _{\eta \rightarrow+0} \frac{-1}{2 \pi i} \int_{0}^{x} d t \int_{C_{\eta t}}(s+t)^{p-1} f^{\prime}(s) d s,
\end{aligned}
$$

where $C_{\eta t}$ is a contour in the cut s-plane from $-t-i \eta$ to $-t+i \eta$.

Hirschman and Widder [3], though mainly concerned with "real" inversion formulae, adapted their convolution transform theory to obtain complex inversion formulae [3, chapter IX]. They deduced in particular [3, page 235] inversion formulae for the transform

$$
f(s)=\int_{-\infty}^{\infty}\left(\frac{1}{2} \operatorname{sech} \frac{s-t}{2}\right)^{p+1} \phi(t) d t,
$$

and (1.1) is easily enough related to this by a logarithmic change of variable followed by an integration by parts. The result is

THEOREM C. If $p>0, \alpha$ is of bounded variation locally in $[0, \infty)$, and (1.1) converges for some $s$ in the cut plane, then for each positive $x$ at which $\alpha$ is continuous

$$
\alpha(x)-\alpha(0)=\lim _{\rho \rightarrow 1-0} \frac{(2 x)^{p}}{4 \pi} \int_{-\pi}^{\pi} f\left(x e^{i \rho y}\right) e^{\frac{1}{2}(p+1) \rho y}\left(\cos \frac{1}{2} y\right)^{p-1} d y .
$$

They also gave a formula, more complicated, for the case $-1<p<0$.

Pandey and Zemanian [4] extended Hirschman and Widder's general complex inversion theory to generalized functions.

Although these various inversion theorems are known, we hope that ours are also worth attention for several reasons. Some of our formulae have something of the simplicity of the original inversion formula (1.2) of Stieltjes and Widder. We prove our formulae for complex $p$ with positive real part, and then in corollaries we give extensions covering all complex $p$ which are not zero or negative integral. Further we show that they hold not only for functions $\alpha$ which are locally of bounded variation, but also for functions locally of bounded $q$ th power variation.

One of our inversion theorems is

THEOREM 6. If re $p>0, \alpha$ is of bounded variation locally in $[0, \infty),(1.1)$ converges for some $s$ in the cut plane, $\lambda>0$ and $x>0$, then

$$
\begin{aligned}
\frac{1}{2}\{\alpha(x+0)+\alpha(x-0)\} & -\alpha(0) \\
& =\lim _{\eta \rightarrow+0} \frac{1}{2 \pi i} \int_{-x}^{\lambda}(x+s)^{p-1}\{f(s-i \eta)-f(s+i \eta)\} d s .
\end{aligned}
$$

Theorem 7 has the same form, except that $\lambda$ is replaced by $\infty$ and the extra hypothesis that $\alpha(t) /\left(1+t^{2}\right) \in L(0, \infty)$ is imposed. This extra hypothesis secures the 
convergence of the infinite integral that (1.6) then contains. We write the upper terminal of this integral (see (7.1)) as $\infty$, not as $\rightarrow \infty$; this is because it is a proper $L$-integral on $(-x, \infty)$, absolutely convergent, not an improper integral defined as a limit of integrals as (1.1) is.

Theorem 4, although more complicated, is the inversion theorem which led us to Theorems 6 and 7. It was found by formal manipulations using fractional integration, which we have thought interesting enough to include as $\$ 2$.

Theorem 5 is an extension of Cauchy's singular integral (also known as one of Plemelj's formulae). Sumner [2, page 179, Lemmas 4a, $4 \mathrm{~b}$ and $4 \mathrm{c}$ ] also uses such a theorem. His is simpler than ours, but ours produces the simpler inversion theorem.

Relations between Sumner's results [2] and ours are discussed in $\S 8$. There is clearly a considerable gap between (1.3) and (1.6), but some approach between other results is found. This discussion also leads to Theorems 8 and 9 , two inversion theorems in our style for the transform

$$
g(s)=\int_{0}^{\rightarrow \infty} \frac{\phi(t)}{(s+t)^{p}} d t .
$$

The introduction of functions $\alpha$ of bounded $q$ th power variation into our theorems is delayed until $\S 9$. There it is shown that the phrase "of bounded variation" can be replaced by "of bounded $q$ th power variation, for $q \geqq 1$ " throughout the paper. The impact of this generalization on the proofs is felt only in $\$ 4$, and much of it is supplied by Theorem 10 which may be thought of as a new theorem of Radon-Nikodym type.

All powers are principal values.

\section{Genesis of our inversion formulae}

Generalized Stieltjes transforms with different values of the parameter $p$ are connected with one another by fractional integration, as is pointed out in [5, page 213]. A case of this will now be shown formally.

If $f$ is the transform (1.1), the $(p-1)$ th Weyl fractional integral of $f$ is

$$
\begin{aligned}
J^{p-1} f(x) & =\int_{x}^{\infty} \frac{(t-x)^{p-2}}{\Gamma(p-1)} f(t) d t=\int_{0}^{\infty} \frac{u^{p-2}}{\Gamma(p-1)} f(x+u) d u \\
& =\int_{0}^{\infty} \frac{u^{p-2}}{\Gamma(p-1)} d u \int_{0}^{\rightarrow \infty} \frac{d \alpha(t)}{(x+u+t)^{p}} \\
& =\frac{1}{\Gamma(p-1)} \int_{0}^{\rightarrow \infty} d \alpha(t) \int_{0}^{\infty} \frac{u^{p-2}}{(x+t+u)^{p}} d u
\end{aligned}
$$

so that, evaluating the inner integral with the help of Lemma 5 below, 


$$
J^{p-1} f(x)=\frac{1}{\Gamma(p)} \int_{0}^{\rightarrow \infty} \frac{d \alpha(t)}{x+t},
$$

which relates the generalized with the ordinary Stieltjes transform.

Inverting (2.2) formally by Theorem A, and using (2.1),

$$
\begin{aligned}
& \frac{1}{2}\{\alpha(x+0)+\alpha(x-0)\}-\frac{1}{2}\{\alpha(+0)+\alpha(0)\} \\
& \quad=\lim _{\eta \rightarrow+0} \frac{\Gamma(p)}{2 \pi i} \int_{-x}^{0} d s \int_{0}^{\infty} \frac{u^{p-2}}{\Gamma(p-1)}\{f(s-i \eta+u)-f(s+i \eta+u)\} d u \\
& \quad=\lim _{\eta \rightarrow+0} \frac{p-1}{2 \pi i} \int_{-x}^{0} d s \int_{s}^{\infty}(t-s)^{p-2}\{f(t-i \eta)-f(t+i \eta)\} d t \\
& \quad=\lim _{\eta \rightarrow+0} \frac{p-1}{2 \pi i} \int_{-x}^{\infty}\{f(t-i \eta)-f(t+i \eta)\} d t \int_{-x}^{\min (0, t)}(t-s)^{p-2} d s \\
& (2.3)=\lim _{\eta \rightarrow+0} \frac{1}{2 \pi i} \int_{-x}^{\infty}\{f(t-i \eta)-f(t+i \eta)\}\left\{(t+x)^{p-1}-t^{p-1} H(t)\right\} d t,
\end{aligned}
$$

where $H(t)$ is Heaviside's unit function

$$
H(t)=0 \text { if } t \leqq 0, \quad H(t)=1 \text { if } t>0 .
$$

We prove (2.3) properly in Theorem 4 . It might be feasible to turn the above formal argument into a proof, but this would involve stricter hypotheses, including re $p>1$. If this were done, there would be little prospect of extending the result to re $p>0$; analytical continuation might seem a natural way, but it is barred by lack of knowledge of uniformity of the limit as $\eta \rightarrow+0$.

\section{Preliminary lemmas}

Several elementary results are needed.

Lemma 1. If $p$ and $z$ are complex numbers, $\mu=$ re $p$ and $v=\operatorname{im} p$, then

$$
|z|^{\mu} e^{-\pi|v|} \leqq\left|z^{p}\right| \leqq|z|^{\mu} e^{\pi|v|}
$$

If $p$ is real or $z$ is positive, the exponential factors may be omitted and equality holds.

Proof. $\left|z^{p}\right|=|\exp (p \log z)|=\exp (\mu \log |z|-v \arg z)=|z|^{\mu} e^{-v \arg z}$.

Lemma 2. If $p, s$ and $s_{0}$ are complex numbers, $\mu=$ re $p$ and $v=\operatorname{im} p$, $M \geqq \max \left(|s|,\left|s_{0}\right|\right)$ and $R \geqq 2 M$, then there is $A=A(p)$, independent of $M, R$, $s$ and $s_{0}$, such that

$$
\left|\frac{\left(s_{0}+R\right)^{p}}{(s+R)^{p}}-1\right| \leqq \frac{A M}{R} .
$$

Proof. Using Lemma 1, 


$$
\begin{aligned}
& \left|(s+R)^{p}\right| \geqq|R+s|^{\mu} e^{-\pi|v|} \geqq(R-|s| \operatorname{sgn} \mu)^{\mu} e^{-\pi|v|} \geqq(R-M \operatorname{sgn} \mu)^{\mu} e^{-\pi|v|}, \\
& \left|\left(s_{0}+R\right)^{p}-(s+R)^{p}\right|=\left|\int_{s}^{s_{0}} p(t+R)^{p-1} d t\right| \leqq\left|s_{0}-s\right||p| \sup _{t}\left|(t+R)^{p-1}\right|
\end{aligned}
$$

where the sup is taken for all $t$ on the line segment from $s$ to $s_{0}$. For such $t$, Lemma 1 gives

$$
\left|(t+R)^{p-1}\right| \leqq|R+t|^{\mu-1} e^{\pi|v|} \leqq(R+M \operatorname{sgn}(\mu-1))^{\mu-1} e^{\pi|v|} .
$$

Together these inequalities give

$$
\begin{aligned}
\left|\frac{\left(s_{0}+R\right)^{p}-(s+R)^{p}}{(s+R)^{p}}\right| & \leqq 2 M|p| e^{2 \pi|v|} \frac{(R+M \operatorname{sgn}(\mu-1))^{\mu-1}}{(R-M \operatorname{sgn} \mu)^{\mu}} \\
& \leqq 2 M|p| e^{2 \pi|v|} \frac{\left(R+\frac{1}{2} R \operatorname{sgn}(\mu-1)\right)^{\mu-1}}{\left(R-\frac{1}{2} R \operatorname{sgn} \mu\right)^{\mu}}
\end{aligned}
$$

The last factor does not exceed $3^{\mu-1} .2 / R$, or $2 / R$, or $3^{-\mu} \cdot 2 / R$, according as $\mu \geqq 1$, or $1>\mu \geqq 0$, or $0>\mu$. This gives the stated inequality, actually with $A=4|p| e^{2 \pi|v|} \max \left(3^{\mu-1}, 1,3^{-\mu}\right)$.

LEMMA 3. If $u$ is real, $\eta$ is positive, $\mu=\operatorname{re} p$ and $v=\operatorname{im} p$, then there is $B=B(p)$, independent of $u$ and $\eta$, such that

$$
\left|(u-i \eta)^{-p-1}-(u+i \eta)^{-p-1}\right| \leqq \begin{cases}B \eta|u|^{-\mu-2} & \text { if } \mu \geqq-2 \\ B \eta|u+i \eta|^{-\mu-2} & \text { if } \mu \leqq-2\end{cases}
$$

Proof. Using Lemma 1,

$$
\begin{aligned}
\left|(u-i \eta)^{-p-1}-(u+i \eta)^{-p-1}\right| & =\left|(p+1) \int_{-\eta}^{\eta}(u+i s)^{-p-2} i d s\right| \\
& \leqq|p+1| \cdot 2 \eta \sup _{-\eta \leqq s \leqq \eta}\left|(u+i s)^{-p-2}\right| \\
& \leqq|p+1| \cdot 2 \eta \sup _{-\eta \leqq s \leqq \eta}|u+i s|^{-\mu-2} e^{\pi|v|} \\
& =2|p+1| e^{\pi|v|} \eta \cdot \max _{\mid}\left\{|u+i \eta|^{-\mu-2},|u|^{-\mu-2}\right\}
\end{aligned}
$$

as required, with $B=2|p+1| e^{\pi|v|}$.

LEMMA 4. If $t$ and $x$ are positive, $\mu=\mathrm{re} p$ and $v=\operatorname{im} p$, then there is $B^{\prime}=B^{\prime}(p)$, independent of $t$ and $x$, such that

$$
\begin{aligned}
& \left|(t+x)^{p-1}-t^{p-1}\right| \leqq\left\{\begin{array}{ll}
\frac{1}{2} B^{\prime} x(t+x)^{\mu-2} & \text { if } \mu \geqq 2, \\
\frac{1}{2} B^{\prime} x t^{\mu-2} & \text { if } \mu \leqq 2 .
\end{array}\right. \text { PROOF. Using Lemma 1, }
\end{aligned}
$$

$$
\begin{aligned}
\left|(t+x)^{p-1}-t^{p-1}\right| & =\left|(p-1) \int_{0}^{x}(t+s)^{p-2} d s\right| \\
& \leqq|p-1| x \sup _{0 \leqq s \leqq x}\left|(t+s)^{p-2}\right|
\end{aligned}
$$




$$
\begin{aligned}
& \leqq|p-1| x \sup _{0 \leqq s \leqq x}|t+s|^{\mu-2} e^{\pi|v|} \\
& =|p-1| e^{\pi|v|} x \max \left\{(t+x)^{\mu-2}, t^{\mu-2}\right\}
\end{aligned}
$$

as required, with $B^{\prime}=2|p-1| e^{\pi|\nu|},=B(-p)$.

LEMMA 5. If $p, a$ and $b$ are complex, $c$ and $x$ are real, $c<x$, and $a+c$ and $b+c$ are neither zero nor negative real, then

$$
\int_{c}^{x} \frac{(b+t)^{p-1}}{(a+t)^{p+1}} d t=\frac{1}{p(a-b)}\left(\frac{(b+x)^{p}}{(a+x)^{p}}-\frac{(b+c)^{p}}{(a+c)^{p}}\right) .
$$

This still holds if $b+c=0$ and re $p>0$.

Proof. This follows from integration of

$$
\frac{(b+t)^{p-1}}{(a+t)^{p+1}}=\frac{1}{p(a-b)}-\frac{d}{d t} \frac{(b+t)^{p}}{(a+t)^{p}},
$$

observing that the left side is continuous in $c \leqq t \leqq x$ because $a+t$ and $b+t$ are neither zero nor negative real there, in the general case.

\section{Fundamental properties of generalized Stieltjes transforms}

These properties are concerned with existence and analyticity of the transform. Theorems 1, 2, 3 are modelled on Widder's Theorems 2a, 2b, 3a [1, pages 326-330].

Throughout the paper $\alpha$ is a complex-valued function defined on $[0, \infty)$.

The generalized Stieltjes transform of $\alpha$ is defined as the "improper" RiemannStieltjes integral

$$
f(s)=\int_{0}^{\rightarrow \infty} \frac{d \alpha(t)}{(s+t)^{p}}=\lim _{R \rightarrow \infty} \int_{0}^{R} \frac{d \alpha(t)}{(s+t)^{p}}
$$

when this limit exists; the "proper"' Riemann-Stieltjes integral in the right hand expression certainly exists for each $R>0$ if $\alpha$ is locally of bounded variation.

THEOREM 1. If $p$ is any complex number, $\alpha$ is of bounded variation locally in $[0, \infty)$, and the integral (1.1) converges for $s=s_{0}$, a complex number in the cut plane omitting the closed negative real axis, then (1.1) converges for every s in this cut plane. Further

$$
f(s)=\int_{0}^{\rightarrow \infty} \frac{d \alpha(t)}{(s+t)^{p}}=f\left(s_{0}\right)+p\left(s_{0}-s\right) \int_{0}^{\infty} \frac{\left(s_{0}+t\right)^{p-1}}{(s+t)^{p+1}} \beta(t) d t
$$

here the integral on the right converges absolutely, and

$$
\beta(t)=\int_{0}^{t} \frac{d \alpha(u)}{\left(s_{0}+u\right)^{p}}
$$

is a function of bounded variation locally in $[0, \infty)$. 
Proof. For any $s$ in the cut plane, $(s+t)^{p}$ is continuous and not zero, for all $t \geqq 0$; and for any $R>0, \alpha(t)$ is of bounded variation on $[0, R]$. So the following integral exists, and

$$
\begin{aligned}
\int_{0}^{R} \frac{d \alpha(t)}{(s+t)^{p}} & =\int_{0}^{R} \frac{\left(s_{0}+t\right)^{p}}{(s+t)^{p}} d \beta(t) \\
& =\frac{\left(s_{0}+R\right)^{p}}{(s+R)^{p}} \beta(R)-\int_{0}^{R} \beta(t) d \frac{\left(s_{0}+t\right)^{p}}{(s+t)^{p}} \\
& =\frac{\left(s_{0}+R\right)^{p}}{(s+R)^{p}} \beta(R)+p\left(s_{0}-s\right) \int_{0}^{R} \beta(t) \frac{\left(s_{0}+t\right)^{p-1}}{(s+t)^{p+1}} d t
\end{aligned}
$$

using standard properties of Stieltjes integrals [1, pages $12,7,11]$.

Again $\beta$ is of bounded variation locally in $[0, \infty)$, as is easily verified, and $\beta(t) \rightarrow f\left(s_{0}\right)$ as $t \rightarrow \infty$. Consequently $\beta(t)$ is bounded on $[0, \infty)$. So also is $\left(s_{0}+t\right)^{p} /(s+t)^{p}$. Thus

$$
\beta(t) \frac{\left(s_{0}+t\right)^{p-1}}{(s+t)^{p+1}}=\frac{\beta(t)}{\left(s_{0}+t\right)(s+t)} \frac{\left(s_{0}+t\right)^{p}}{(s+t)^{p}}=O\left(\frac{1}{t^{2}}\right)
$$

as $t \rightarrow \infty$, and so the integral on the right of (4.4) converges as $R \rightarrow \infty$, indeed absolutely. The other term on the right of (4.4) also converges, to $f\left(s_{0}\right)$; this gives (4.1) and completes the proof.

Definition. We say that the integral (1.1) "converges" if it converges for some s in the cut plane omitting the closed negative real axis. Under the conditions of Theorem 1 it then converges for all such $s$.

THEOREM 2. If $p$ is any complex number, $\alpha$ is of bounded variation locally in $[0, \infty)$, and the integral (1.1) converges, then it converges uniformly on each bounded closed subset of the cut s-plane.

Proor. Define $s_{0}$ and $\beta(t)$ as in the statement of Theorem 1. Suppose that $s$ is in the cut plane, and that $R>0$. Subtracting (4.4) from (4.1),

$$
\begin{aligned}
\int_{R}^{\rightarrow \infty} \frac{d \alpha(t)}{(s+t)^{p}}= & f\left(s_{0}\right)-\frac{\left(s_{0}+R\right)^{p}}{(s+R)^{p}} \beta(R)+p\left(s_{0}-s\right) \int_{R}^{\infty} \frac{\left(s_{0}+t\right)^{p-1}}{(s+t)^{p+1}} \beta(t) d t \\
= & f\left(s_{0}\right)-\beta(R)+\left(1-\frac{\left(s_{0}+R\right)^{p}}{(s+R)^{p}}\right) \beta(R) \\
& \quad+p\left(s_{0}-s\right) \int_{R}^{\infty} \frac{\left(s_{0}+t\right)^{p}}{(s+t)^{p}} \frac{\beta(t)}{(s+t)\left(s_{0}+t\right)} d t
\end{aligned}
$$

Let $M$ be the radius of a neighbourhood of the origin containing the given bounded closed set $Q$ and also containing $s_{0}$. Suppose that $R \geqq 2 M$ and that $s \in Q$; 
then Lemma 2 is applicable. Also there is $K$ such that $|\beta(t)| \leqq K$, as shown in the proof of Theorem 1. Thus (4.5) gives

$$
\begin{aligned}
& \left|\int_{R}^{\rightarrow \infty} \frac{d \alpha(t)}{(s+t)^{p}}\right| \leqq\left|f\left(s_{0}\right)-\beta(R)\right|+\frac{A M K}{R} \\
& \quad+\left|p\left(s_{0}-s\right)\right| \int_{R}^{\infty}\left(1+\frac{A M}{t}\right) \frac{K}{|s+t|\left|s_{0}+t\right|} d t \\
& \leqq\left|\int_{R}^{\rightarrow \infty} \frac{d \alpha(t)}{\left(s_{0}+t\right)^{p}}\right|+\frac{A M K}{R}+2 M|p|\left(1+\frac{A M}{R}\right) \int_{R}^{\infty} \frac{K}{(t-M)^{2}} d t
\end{aligned}
$$

This last expression is independent of $s$ in $Q$, and tends to 0 as $R \rightarrow \infty$; which proves the required uniform convergence on $Q$.

COROLlary 2. If $p$ is complex, $\alpha$ is locally of bounded variation, and the integral

$$
f(s)=\int_{0}^{\rightarrow \infty} \frac{d \alpha(t)}{(s+t)^{p}}
$$

converges, then $f$ is regular in the cut plane. Its derivatives are correctly given by formal differentiation through the integral sign, thus:

$$
f^{(m)}(s)=(-1)^{m}(p)_{m} \int_{0}^{\rightarrow \infty} \frac{d \alpha(t)}{(s+t)^{p+m}}
$$

for all positive integers $m$ and all $s$ in the cut plane; where

$$
(p)_{m}=p(p+1) \cdots(p+m-1) .
$$

THEOREM 3. If $\mathrm{re} p>0, \alpha$ is of bounded variation locally in $[0, \infty)$, and the integral

$$
f(s)=\int_{0}^{\rightarrow \infty} \frac{d \alpha(t)}{(s+t)^{p}}
$$

converges, then

$$
\alpha(t)=o\left(t^{p}\right) \text { as } t \rightarrow \infty .
$$

Proof. As in (4.2) with $s_{0}=1$, let $\beta$ be the function

$$
\beta(t)=\int_{0}^{t} \frac{d \alpha(u)}{(1+u)^{p}} \quad \text { for } t \geqq 0 .
$$

By Theorem 1, $f(1)$ exists; so $\beta(t) \rightarrow f(1)$ as $t \rightarrow \infty$. Writing $\beta(\infty)$ for $f(1)$,

$$
\alpha(t)-\alpha(0)=\int_{0}^{t} \frac{(1+u)^{p}}{(1+u)^{p}} d \alpha(u)=\int_{0}^{t}(1+u)^{p} d \beta(u)
$$




$$
\begin{aligned}
= & (1+t)^{p} \beta(t)-p \int_{0}^{t}(1+u)^{p-1} \beta(u) d u \\
= & (1+t)^{p}\{\beta(t)-\beta(\infty)\}+\beta(\infty)+p \int_{0}^{t}(1+u)^{p-1}\{\beta(\infty)-\beta(u)\} d u ; \\
& \frac{\alpha(t)}{t^{p}}=\frac{\alpha(0)+\beta(\infty)}{t^{p}}+\frac{(1+t)^{p}}{t^{p}}\{\beta(t)-\beta(\infty)\}+I
\end{aligned}
$$

where

$$
I=\frac{p}{t^{p}} \int_{0}^{t}(1+u)^{p-1}\{\beta(\infty)-\beta(u)\} d u .
$$

It is clear that the first two terms on the right of (4.9) tend to 0 as $t \rightarrow \infty$, using the datum that re $p>0$ for the first time. It remains only to prove that $I$, given by (4.10), tends to 0 .

Given $\varepsilon>0$ there is $m$ such that

$$
|\beta(\infty)-\beta(u)|<\varepsilon \text { for all } u \geqq m .
$$

Since also $\beta$ is locally of bounded variation, it is globally bounded: there is $M$ such that

$$
|\beta(\infty)-\beta(u)|<M \text { for all } u \geqq 0 \text {. }
$$

Writing $\mu=$ re $p$, these with (4.10) give, for $t \geqq m$,

$$
\begin{aligned}
|I| & \leqq \frac{|p|}{t^{\mu}} \int_{0}^{t}(1+u)^{\mu-1}|\beta(\infty)-\beta(u)| d u \\
& \leqq \frac{|p|}{t^{\mu}}\left(\int_{0}^{m}(1+u)^{\mu-1} M d u+\int_{m}^{t}(1+u)^{\mu-1} \varepsilon d u\right) \\
& =\frac{|p|}{\mu t^{\mu}}\left[M\left\{(1+m)^{\mu}-1\right\}+\varepsilon\left\{(1+t)^{\mu}-(1+m)^{\mu}\right\}\right] \\
& <\frac{|p|}{\mu}\left(M \frac{(1+m)^{\mu}}{t^{\mu}}+\varepsilon \frac{(1+t)^{\mu}}{t^{\mu}}\right) .
\end{aligned}
$$

It follows that

$$
\varlimsup_{t \rightarrow \infty}|I| \leqq \frac{|p|}{\mu} \varepsilon
$$

for every $\varepsilon>0$; consequently $I \rightarrow 0$ as $t \rightarrow \infty$, as required.

COROLlaRY 3. If re $p>0, \alpha(0)=0, \alpha$ is of bounded variation locally in $[0, \infty)$, and the integral (1.1) converges, then for all $s$ in the cut plane

$$
f(s)=\int_{0}^{\rightarrow \infty} \frac{p \alpha(t)}{(s+t)^{p+1}} d t .
$$


Proof. This follows from (1.1) by integration by parts, the integrated part vanishing at the lower terminal by data and at the upper terminal by Theorem 3 .

\section{The first inversion formula}

We now prove a theorem suggested by the formal result of $\$ 2$.

THEOREM 4. If re $p>0, \alpha$ is of bounded variation locally in $[0, \infty)$ $\alpha(t) /\left(1+t^{3}\right) \in L(0, \infty)$ and the integral

$$
f(s)=\int_{0}^{\infty} \frac{d \alpha(t)}{(s+t)^{p}}
$$

converges, then for each positive $x$

$$
\begin{aligned}
& \frac{1}{2}\{\alpha(x+0)+\alpha(x-0)\}-\frac{1}{2}\{\alpha(+0)+\alpha(0)\} \\
& \quad=\lim _{\eta \rightarrow+0} \frac{1}{2 \pi i} \int_{-x}^{\infty}\left\{(t+x)^{p-1}-t^{p-1} H(t)\right\}\{f(t-i \eta)-f(t+i \eta)\} d t,
\end{aligned}
$$

where $H(t)$ is Heaviside's unit function and the integral in (5.1) is absolutely convergent.

Proof. (i) We first suppose that $\alpha(0)=0$. By Corollary $2, f$ is continuous whenever it is used in (5.1), and in the integrals $E_{1}$ and $E_{2}$ defined below. The double integrals (5.2) and (5.4) will be shown in (ii) to be absolutely convergent, and this will incidentally establish the existence of $E_{1}$ and $E_{2}$ with which we start.

$$
\begin{aligned}
E_{1} & =\int_{0}^{\infty}\left\{(t+x)^{p-1}-t^{p-1}\right\}\{f(t-i \eta)-f(t+i \eta)\} d t \\
& =\int_{0}^{\infty}\left\{(t+x)^{p-1}-t^{p-1}\right\} d t \int_{0}^{\rightarrow \infty}\left(\frac{1}{(u+t-i \eta)^{p+1}}-\frac{1}{(u+t+i \eta)^{p+1}}\right) p \alpha(u) d u
\end{aligned}
$$

using Corollary 3. Assuming the absolute convergence of the inner integral and the correctness of changing the order of integration, which will follow from (ii), and using Lemma 5 to evaluate the resulting inner integral,

$$
\begin{aligned}
E_{1}= & \int_{0}^{\infty} p x(u) d u \int_{0}^{\infty}\left\{(t+x)^{p-1}-t^{p-1}\right\}\left(\frac{1}{(u+t-i \eta)^{p+1}}-\frac{1}{(u+t+i \eta)^{p+1}}\right) d t \\
= & \int_{0}^{\infty}\left\{\frac{1}{u-i \eta-x}\left(1-\frac{x^{p}}{(u-i \eta)^{p}}\right)-\frac{1}{u-i \eta}\right. \\
& \left.-\frac{1}{u+i \eta-x}\left(1-\frac{x^{p}}{(u+i \eta)^{p}}\right)+\frac{1}{u+i \eta}\right\} \alpha(u) d u
\end{aligned}
$$


A very similar treatment of $E_{2}$ runs as follows.

$$
\begin{aligned}
E_{2} & =\int_{-x}^{0}(t+x)^{p-1}\{f(t-i \eta)-f(t+i \eta)\} d t \\
& =\int_{-x}^{0}(t+x)^{p-1} d t \int_{0}^{\rightarrow \infty}\left(\frac{1}{(u+t-i \eta)^{p+1}}-\frac{1}{(u+t+i \eta)^{p+1}}\right) p \alpha(u) d u \\
& =\int_{0}^{\infty} p x(u) d u \int_{-x}^{\iota}(t+x)^{p-1}\left(\frac{1}{(u+t-i \eta)^{p+1}}-\frac{1}{(u+t+i \eta)^{p+1}}\right) d t \\
& =\int_{0}^{\infty}\left(\frac{1}{u-i \eta-x} \frac{x^{p}}{(u-i \eta)^{p}}-\frac{1}{u+i \eta-x} \frac{x^{p}}{(u+i \eta)^{p}}\right) \alpha(u) d u
\end{aligned}
$$

Combining (5.3) and (5.5),

$$
\frac{E_{1}+E_{2}}{2 \pi i}=\frac{1}{2 \pi i} \int_{0}^{\infty}\left(\frac{1}{u-x-i \eta}-\frac{1}{u-x+i \eta}-\frac{1}{u-i \eta}+\frac{1}{u+i \eta}\right) \alpha(u) d u
$$

$$
\begin{aligned}
& =\frac{1}{\pi} \int_{0}^{\infty}\left(\frac{\eta}{(u-x)^{2}+\eta^{2}}-\frac{\eta}{u^{2}+\eta^{2}}\right) \alpha(u) d u \\
& =\frac{1}{\pi} \int_{0}^{R}\left(\frac{\eta}{(u-x)^{2}+\eta^{2}}-\frac{\eta}{u^{2}+\eta^{2}}\right) \alpha(u) d u+E
\end{aligned}
$$

where $R$ is chosen greater than $x$, and

$$
\begin{aligned}
|E| & =\left|\frac{\eta}{\pi} \int_{R}^{\infty} \frac{x(2 u-x)}{\left((u-x)^{2}+\eta^{2}\right)\left(u^{2}+\eta^{2}\right)} \alpha(u) d u\right| \\
& \leqq \frac{\eta}{\pi} \int_{R}^{\infty} \frac{2 x u}{(u-x)^{2} u^{2}}|\alpha(u)| d u \rightarrow 0 \text { as } \eta \rightarrow+0,
\end{aligned}
$$

the integral being convergent by the integrability hypothesis, and independent of $\eta$. Plemelj's formula applied to the first part of (5.7) now gives, if $x>0$,

$$
\left(E_{1}+E_{2}\right) / 2 \pi i \rightarrow \frac{1}{2}\{\alpha(x+0)+\alpha(x-0)\}-\frac{1}{2} \alpha(+0) \text { as } \eta \rightarrow+0 .
$$

Replacing $\alpha(x)$ by $\alpha(x)-\alpha(0)$, to remove the restriction $\alpha(0)=0$, we now have (5.1), subject to the provisos mentioned.

It should be noticed that (5.6) cannot be separated into two integrals to each of which Plemelj's formula might be applied, because the separate infinite integrals might be divergent. However this separation could be made if the integrability hypothesis were stiffened to $\alpha(t) /\left(1+t^{2}\right) \in L(0, \infty)$.

(ii) We now verify the provisos assumed above, namely the absolute convergence of the double integrals (5.2) and (5.4). To do this requires separate treatment of various ranges of integration, and we consider four double integrals $K_{1}(x)$, $K_{2}(x), K_{3}$ and $K_{4}$. 
Choose $R>x \geqq 0, \eta>0$ and $\lambda>0$. Let $\mu=\operatorname{re} p$ and $v=\operatorname{im} p$.

$$
\begin{aligned}
K_{1}(x) & =\int_{0}^{R}|\alpha(u)| d u \int_{0}^{\lambda}\left|(t+x)^{p-1}\left(\frac{1}{(u+t-i \eta)^{p+1}}-\frac{1}{(u+t+i \eta)^{p+1}}\right)\right| d t \\
& \leqq \int_{0}^{R}|\alpha(u)| d u \int_{0}^{\lambda}(t+x)^{\mu-1} \frac{2 e^{\pi|v|}}{|u+t+i \eta|^{\mu+1}} d t, \quad \text { by Lemma } 1, \\
\text { (5.8) } & \leqq \frac{2 e^{\pi|v|}}{\eta^{\mu+1}} \int_{0}^{R}|\alpha(u)| d u \int_{0}^{\lambda}(t+x)^{\mu-1} d t ;
\end{aligned}
$$

this is finite because $\alpha$ is locally bounded, $\eta>0, x \geqq 0$ and $\mu>0$.

$$
\begin{aligned}
K_{2}(x) & =\int_{R}^{\infty}|\alpha(u)| d u \int_{0}^{\lambda}\left|(t+x)^{p-1}\left(\frac{1}{(u+t-i \eta)^{p+1}}-\frac{1}{(u+t+i \eta)^{p+1}}\right)\right| d t \\
& \leqq \int_{R}^{\infty}|\alpha(u)| d u \int_{0}^{\lambda}(t+x)^{\mu-1} \frac{B \eta}{(u+t)^{\mu+2}} d t, \quad \text { by Lemma 3, } \\
(5.9) & \leqq B \eta \int_{R}^{\infty} \frac{|\alpha(u)|}{u^{\mu+2}} d u \int_{0}^{\lambda}(t+x)^{\mu-1} d t ;
\end{aligned}
$$

this is finite because $\alpha(u)=O\left(u^{\mu}\right)$ as $u \rightarrow \infty$, by Theorem 3 , and because $x \geqq 0$ and $\mu>0$.

$$
\begin{aligned}
K_{3} & =\int_{0}^{R}|\alpha(u)| d u \int_{\lambda}^{\infty}\left|(t+x)^{p-1}-t^{p-1}\right|\left|\frac{1}{(u+t-i \eta)^{p+1}}-\frac{1}{(u+t+i \eta)^{p+1}}\right| d t \\
& \leqq \int_{0}^{R}|\alpha(u)| d u \int_{\lambda}^{\infty}\left\{(t+x)^{\mu-1}+t^{\mu-1}\right\} \frac{B \eta}{(u+t)^{\mu+2}} d t, \quad \text { by Lemma 3, } \\
& \leqq B \eta \int_{0}^{R}|\alpha(u)| d u \int_{\lambda}^{\infty} \frac{(t+x)^{\mu-1}+t^{\mu-1}}{t^{\mu+2}} d t ;
\end{aligned}
$$

this is finite because $\alpha$ is locally bounded and $\lambda>0$.

$$
\begin{gathered}
K_{4}=\int_{R}^{\infty}|\alpha(u)| d u \int_{\lambda}^{\infty}\left|(t+x)^{p-1}-t^{p-1}\right|\left|\frac{1}{(u+t-i \eta)^{p+1}}-\frac{1}{(u+t+i \eta)^{p+1}}\right| d t \\
\text { (5.10) } \leqq \int_{R}^{\infty}|\alpha(u)| d u \int_{2}^{\infty} \frac{1}{2} B^{\prime} x(t+x)^{\mu-2} \frac{B \eta}{(u+t)^{\mu+2}} d t,
\end{gathered}
$$

supposing that $\mu \geqq 2$, and using Lemmas 3 and 4 ; and, since also $0 \leqq x<R \leqq u$,

$$
\begin{aligned}
K_{4} & \leqq \frac{1}{2} B^{\prime} B \eta x \int_{R}^{\infty}|\alpha(u)| d u \int_{\lambda}^{\infty} \frac{(t+u)^{\mu-2}}{(t+u)^{\mu+2}} d t \\
& =\frac{1}{6} B^{\prime} B \eta x \int_{R}^{\infty} \frac{|\alpha(u)|}{(\lambda+u)^{3}} d u<\infty .
\end{aligned}
$$


If $1<\mu<2$ the application of Lemma 4 is different, and (5.10) is replaced by

$$
\begin{aligned}
K_{4} & \leqq \int_{R}^{\infty}|\alpha(u)| d u \int_{2}^{\infty} \frac{1}{2} B^{\prime} x t^{\mu-2} \frac{B \eta}{(u+t)^{\mu+2}} d t \\
& =\frac{1}{2} B^{\prime} B \eta x \int_{R}^{\infty} \frac{|\alpha(u)|}{u^{3}} d u \int_{\lambda / u}^{\infty} \frac{v^{\mu-2}}{(1+v)^{\mu+2}} d v \quad \text { putting } t=u v, \\
& \leqq \frac{1}{2} B^{\prime} B \eta x \int_{R}^{\infty} \frac{|\alpha(u)|}{u^{3}} d u \int_{0}^{\infty} \frac{v^{\mu-2}}{(1+v)^{\mu+2}} d v<\infty
\end{aligned}
$$

If $0<\mu \leqq 1$ the inequality (5.11) is still obtained as above, but it needs a different treatment, such as

$$
\begin{aligned}
K_{4} & \leqq \frac{1}{2} B^{\prime} B \eta x \int_{R}^{\infty}|\alpha(u)| d u \int_{\lambda}^{\infty} \frac{t^{\mu-2}}{(u+\lambda)^{\mu+3 / 2}(R+t)^{\frac{1}{2}}} d t \\
& \leqq \frac{1}{2} B^{\prime} B \eta x \int_{R}^{\infty} \frac{|\alpha(u)|}{u^{\mu+\overline{3} / 2}} d u \int_{\lambda}^{\infty} t^{\mu-5 / 2} d t
\end{aligned}
$$

this is finite because $\alpha(u)=O\left(u^{\mu}\right)$ as $u \rightarrow \infty$, by Theorem 3 , and because $5 / 2-\mu>1$. Thus $K_{4}<\infty$ for all admissible values of $\mu$.

For the absolute convergence of (5.2) we now have

$$
\begin{gathered}
\int_{0}^{\infty}|\alpha(u)| d u \int_{0}^{\infty}\left|(t+x)^{p-1}-t^{p-1}\right|\left|\frac{1}{(u+t-i \eta)^{p+1}}-\frac{1}{(u+t+i \eta)^{p+1}}\right| d t \\
\leqq\left\{K_{1}(x)+K_{1}(0)\right\}+\left\{K_{2}(x)+K_{2}(0)\right\}+K_{3}+K_{4}<\infty .
\end{gathered}
$$

For the absolute convergence of (5.4) we need only observe that the sequences of inequalities ending in (5.8) and (5.9) still stand if the terminals of the inner integrals are changed throughout from 0 and $\lambda$ to $-x$ and 0 respectively.

This completes the proof of Theorem 4 .

Remark. When $p=1$, Theorem 4 reduces to Theorem $A$. For suppose the hypotheses of Theorem $A$ hold. Then Theorem 3 gives that $\alpha(t)=o(t)$, whence $\alpha(t) /\left(1+t^{3}\right) \in L(0, \infty)$. So all hypotheses of Theorem 4 hold. Its conclusion (5.1) therefore also holds; and this reduces to (1.2) because

$$
(t+x)^{0}-t^{0} H(t)= \begin{cases}0 & \text { if } t>0 \\ 1 & \text { if } \quad-x<t \leqq 0 .\end{cases}
$$

Corollary 4. If re $p>-n$, where $n$ is a positive integer and $p$ is not zero or a negative integer, and if the other hypotheses of Theorem 4 hold, then for each positive $x$

$$
\begin{aligned}
& \frac{1}{2}\{\alpha(x+0)+\alpha(x-0)\}-\frac{1}{2}\{\alpha(+0)+\alpha(0)\} \\
& \quad=\lim _{\eta \rightarrow+0} \frac{(-1)^{n}}{2 \pi i(p)_{n}} \int_{-x}^{\infty}\left\{(t+x)^{p+n-1}-t^{p+n-1} H(t)\right\}\left\{f^{(n)}(t-i \eta)-f^{(n)}(t+i \eta)\right\} d t,
\end{aligned}
$$


where $(p)_{n}$ has the meaning assigned in (4.7) and $H(t)$ is Heaviside's unit function.

Proof. Since $(p)_{n} \neq 0,(4.6)$ and Corollary 2 give

$$
\frac{f^{(n)}(s)}{(-1)^{n}(p)_{n}}=\int_{0}^{\rightarrow \infty} \frac{d \alpha(t)}{(s+t)^{p+n}} .
$$

Our conclusion follows by inverting this, using Theorem 4 with $p$ and $f(s)$ replaced by $p+n$ and the left side of (5.12).

\section{A finite-integral inversion formula}

The treatment of $E_{1}$ and $E_{2}$ in the early paragraphs of the proof of Theorem 4 suggests that manageable expressions in terms of $\alpha$ are also obtainable by the same method, at least formally, for such integrals as

$$
\int_{0}^{\infty} h_{p}(x, t, \eta) d t, \quad \int_{0}^{\lambda} h_{p}(x, t, \eta) d t, \quad \int_{-x}^{\lambda} h_{p}(x, t, \eta) d t,
$$

where

$$
h_{p}(x, t, \eta)=(x+t)^{p-1}\{f(t-i \eta)-f(t+i \eta)\}
$$

The simplest of these is the third; it is considered in Lemma 6 below. The other two give expressions of the same general form, but with integrands involving four terms instead of two.

We are thus led to study the behaviour of the right side of (6.1) as $\eta \rightarrow 0$. Theorem 5 below shows that this is especially simple, the kernel approximating the delta function as $\eta \rightarrow+0$ (apart from a constant multiplier). This leads to another inversion theorem, Theorem 6 . This involves a finite range of integration instead of the infinite one in Theorem 4, and does not require the global integrability hypothesis of that theorem.

Lemmas 7 and 8 and Theorem 5 establish the delta function property mentioned, and constitute a partial digression from our main thesis in that they contain no specific reference to Stieltjes transforms.

LEMMA 6. If re $p>0, \alpha$ is of bounded variation locally in $[0, \infty), \alpha(0)=0$, the integral (1.1) defining $f$ converges, and $\eta>0$, then for each $x \geqq 0$ and $\lambda>-x$

$$
\begin{gathered}
\int_{-x}^{\lambda}(x+t)^{p-1}\{f(t-i \eta)-f(t+i \eta)\} d t \\
=\int_{0}^{\infty}\left(\frac{1}{u-x-i \eta} \frac{(x+\lambda)^{p}}{(u+\lambda-i \eta)^{p}}-\frac{1}{u-x+i \eta} \frac{(x+\lambda)^{p}}{(u+\lambda+i \eta)^{p}}\right) \alpha(u) d u .
\end{gathered}
$$


Proof. The integral on the left of (6.1) is $E_{2}$ in the proof of Theorem 4, except that the upper terminal is $\lambda$ instead of 0 . Applying the same treatment as gives (5.5) in that proof, using Corollaries 2 and 3 and Lemma 5, we obtain (6.1) subject to proving the absolute convergence of (compare (5.4))

$$
\int_{0}^{\infty} \alpha(u) d u \int_{-x}^{\lambda}(x+t)^{p-1}\left(\frac{1}{(u+t-i \eta)^{p+1}}-\frac{1}{(u+t+i \eta)^{p+1}}\right) d t .
$$

This absolute convergence is proved by exactly the same treatment as $K_{1}(x)$ and $K_{2}(x)$ are given in (5.8) and (5.9), the only differences being that the lower terminals of all the inner integrals are changed from 0 to $-x$ and the denominator factor $u^{\mu+2}$ in (5.9) is replaced by $(u-x)^{\mu+2}$.

LEMMA 7. If $p$ is any complex number, $\phi(u) /(1+u)^{p+2} \in L(0, \infty), x>-\lambda$ and $R>x \geqq 0$, then as $\eta \rightarrow 0$,

$\int_{R}^{\infty}\left(\frac{1}{u-x-i \eta} \frac{1}{(u+\lambda-i \eta)^{p}}-\frac{1}{u-x+i \eta} \frac{1}{(u+\lambda+i \eta)^{p}}\right) \phi(u) d u=O(\eta)$.

Proof. Let $G$ denote the integrand omitting the factor $\phi(u)$; then

$$
\begin{gathered}
G=\left(\frac{1}{u-x-i \eta}-\frac{1}{u-x}\right) \frac{1}{(u+\lambda-i \eta)^{p}}-\left(\frac{1}{u-x+i \eta}-\frac{1}{u-x}\right) \frac{1}{(u+\lambda+i \eta)^{p}} \\
+\frac{1}{u-x}\left(\frac{1}{(u+\lambda-i \eta)^{p}}-\frac{1}{(u+\lambda+i \eta)^{p}}\right) .
\end{gathered}
$$

Also let $\mu=$ re $p$ and $\nu=$ im $p$, and suppose $\eta>0$ as we may.

(i) Suppose that $\mu \geqq-1$. Lemmas 1 and 3 give

$|G| \leqq \frac{\eta}{|u-x-i \eta||u-x|} \frac{e^{\pi|v|}}{|u+\lambda-i \eta|^{\mu}}+\frac{\eta}{|u-x+i \eta||u-x|} \frac{e^{\pi|v|}}{|u+\lambda+i \eta|^{\mu}}$

$$
+\frac{1}{|u-x|} \frac{2|p| e^{\pi|v|} \eta}{|u+\lambda|^{\mu+1}}
$$

$$
=\left|\frac{u+\lambda-i \eta}{u-x-i \eta}\right| \frac{2 e^{\pi|v|} \eta}{|u-x||u+\lambda-i \eta|^{\mu+1}}+\frac{2|p| e^{\pi|v|} \eta}{|u-x||u+\lambda|^{\mu+1}} .
$$

Also, if $u \geqq R$,

and

$$
\left|\frac{u+\lambda-i \eta}{u-x-i \eta}\right|=\left|1+\frac{\lambda+x}{u-x-i \eta}\right| \leqq 1+\frac{\lambda+x}{u-x} \leqq 1+\frac{\lambda+x}{R-x}=\frac{R+\lambda}{R-x}
$$

$$
|G| \leqq\left(\frac{R+\lambda}{R-x}+|p|\right) \frac{2 e^{\pi|v|} \eta}{(u-x)(u+\lambda)^{\mu+1}} .
$$


Thus

$$
\begin{aligned}
\left|\int_{R}^{\infty} G \phi(u) d u\right| & \leqq\left(\frac{R+\lambda}{R-x}+|p|\right) 2 e^{\pi|v|} \eta \int_{R}^{\infty} \frac{|\phi(u)|}{(u-x)^{\mu+2}} d u, \\
& =O(\eta) \text { as } \eta \rightarrow 0, \text { as required. }
\end{aligned}
$$

(ii) If $\mu<-1$, the same treatment applies, except that the last denominator factor $|u+\lambda|^{\mu+1}$ in (6.2), (6.3) and (6.4) must be changed to $|u+\lambda-i \eta|^{\mu+1}$, and a corresponding minor change occurs in the final inequality.

Lemma 8. If $p$ is any complex number, $\phi \in L(0, R), \lambda>0, R>x>0$ and the limits $\phi(x \pm 0)$ exist, then

$$
\begin{aligned}
\frac{1}{2 \pi i} \int_{0}^{R}\left(\frac{1}{u-x-i \eta} \frac{(x+\lambda)^{p}}{(u+\lambda-i \eta)^{p}}-\frac{1}{u-x+i \eta} \frac{(x+\lambda)^{p}}{(u+\lambda+i \eta)^{p}}\right) \phi(u) d u \\
\rightarrow \frac{1}{2}\{\phi(x+0)+\phi(x-0)\} \quad \text { as } \eta \rightarrow+0 .
\end{aligned}
$$

If $x=0$ this still holds with $\phi(x-0)$ replaced by 0 .

Proof. The integral exists for every $\eta>0$, because the pre-factor of $\phi(u)$ in the integrand is a continuous function of $u$. Denote this pre-factor by $H$. Similarly the following integral $I$ exists.

$$
\begin{aligned}
I & =\int_{0}^{R}\left\{\left(\frac{1}{u-x-i \eta}-\frac{1}{u-x+i \eta}\right)-H\right\} \phi(u) d u \\
& =\int_{0}^{R}\left\{\frac{1}{u-x-i \eta}\left(1-\frac{(x+\lambda)^{p}}{(u+\lambda-i \eta)^{p}}\right)-\frac{1}{u-x+i \eta}\left(1-\frac{(x+\lambda)^{p}}{(u+\lambda+i \eta)^{p}}\right)\right\} \phi(u) d u \\
& =\int_{0}^{R}\left(\int_{\lambda}^{\infty} \frac{p(x+t)^{p-1}}{(u+t-i \eta)^{p+1}} d t-\int_{\lambda}^{\infty} \frac{p(x+t)^{p-1}}{(u+t+i \eta)^{p+1}} d t\right) \phi(u) d u \\
& =\int_{0}^{R} p \phi(u) d u \int_{\lambda}^{\infty}(x+t)^{p-1}\left(\frac{1}{(u+t-i \eta)^{p+1}}-\frac{1}{(u+t+i \eta)^{p+1}}\right) d t
\end{aligned}
$$

here we have used Lemma 5.

Now using Lemma 3, and supposing that $\mu=$ re $p \leqq-2$,

$$
\begin{aligned}
|I| & \leqq|p| \int_{0}^{R}|\phi(u)| d u \int_{\lambda}^{\infty}(x+t)^{\mu-1} \frac{B \eta}{\left((u+t)^{2}+\eta^{2}\right)^{\frac{1}{2}(\mu+2)}} d t \\
& \leqq|p| B \eta \int_{0}^{R}|\phi(u)| d u \int_{\lambda}^{\infty}(x+t)^{\mu-1}\left\{(R+t)^{2}+(R+\lambda)^{2}\right\}^{-\frac{1}{2}(\mu+2)} d t
\end{aligned}
$$

provided that $0<\eta \leqq R+\lambda$; and so, as $\eta \rightarrow+0$,

$$
|I| \leqq|p| B \eta \int_{0}^{R}|\phi(u)| d u \int_{\lambda}^{\infty} 2^{-\frac{1}{2}(\mu+2)}(x+t)^{\mu-1}(R+t)^{-\mu-2} d t=O(\eta) .
$$


If on the other hand $\mu>-2$, these inequalities are replaced by

$$
\begin{aligned}
|I| & \leqq|p| \int_{0}^{R}|\phi(u)| d u \int_{\lambda}^{\infty}(x+t)^{\mu-1} \frac{B \eta}{(u+t)^{\mu+2}} d t \\
& \leqq|p| B \eta \int_{0}^{R}|\phi(u)| d u \int_{\lambda}^{\infty} \frac{(x+t)^{\mu-1}}{t^{\mu+2}} d t=O(\eta) .
\end{aligned}
$$

Whatever the value of $p$, then, $I=O(\eta)$ as $\eta \rightarrow+0$. Consequently

$$
\begin{aligned}
\frac{1}{2 \pi i} \int_{0}^{R} H \phi(u) d u & =\frac{1}{2 \pi i} \int_{0}^{R}\left(\frac{1}{u-x-i \eta}-\frac{1}{u-x+i \eta}\right) \phi(u) d u-\frac{I}{2 \pi i} \\
& =\frac{1}{\pi} \int_{0}^{R} \frac{\eta}{(u-x)^{2}+\eta^{2}} \phi(u) d u+O(\eta) \\
& \rightarrow \frac{1}{2}\{\phi(x+0)+\phi(x-0)\} \quad \text { as } \eta \rightarrow+0,
\end{aligned}
$$

by Cauchy's singular integral $[6$, page 30$]$, which also gives the modification when $x=0$.

THEOREM 5. If $p$ is any complex number, $\phi(u) /(1+u)^{p+2} \in L(0, \infty), \lambda>0$, $x>0$ and the limits $\phi(x \pm 0)$ exist, then

$$
\begin{aligned}
& \frac{1}{2 \pi i} \int_{0}^{\infty}\left(\frac{1}{u-x-i \eta} \frac{(x+\lambda)^{p}}{(u+\lambda-i \eta)^{p}}-\frac{1}{u-x+i \eta} \frac{(x+\lambda)^{p}}{(u+\lambda+i \eta)^{p}}\right) \phi(u) d u \\
& \rightarrow \frac{1}{2}\{\phi(x+0)+\phi(x-0)\} \quad \text { as } \eta \rightarrow+0 .
\end{aligned}
$$

If $x=0$ this still holds with $\phi(x-0)$ replaced by 0 .

Proof. Choosing $R>x$, Lemmas 7 and 8 give this immediately.

Remark. When $p=0$ Lemma 8 and Theorem 5 reduce to Cauchy's singular integral for a finite [1, page 338] and a semi-infinite [6, page 30] interval respectively, with the standard hypotheses.

THEOREM 6. If re $p>0, \alpha$ is of bounded variation locally in $[0, \infty)$, the integral

$$
f(s)=\int_{0}^{\rightarrow \infty} \frac{d \alpha(t)}{(s+t)^{p}}
$$

converges, and $\lambda>0$, then for each positive $x$

$$
\begin{aligned}
\frac{1}{2}\{\alpha(x+0)+\alpha(x-0)\}-\alpha(0) \\
=\lim _{\eta \rightarrow+0} \frac{1}{2 \pi i} \int_{-x}^{\lambda}(x+t)^{p-1}\{f(t-i \eta)-f(t+i \eta)\} d t .
\end{aligned}
$$

If $x=0$ this still holds with $\alpha(-0)$ replaced by $\alpha(0)$. 
Proof. First suppose that $\alpha(0)=0$. By Lemma 6,

$$
\begin{aligned}
E & =\frac{1}{2 \pi i} \int_{-x}^{\lambda}(x+t)^{p-1}\{f(t-i \eta)-f(t+i \eta)\} d t \\
& =\frac{1}{2 \pi i} \int_{0}^{\infty}\left(\frac{1}{u-x-i \eta} \frac{(x+\lambda)^{p}}{(u+\lambda-i \eta)^{p}}-\frac{1}{u-x+i \eta} \frac{(x+\lambda)^{p}}{(u+\lambda+i \eta)^{p}}\right) \alpha(u) d u .
\end{aligned}
$$

By Theorem 3, $\alpha(u)=o\left(u^{\mu}\right)$ as $u \rightarrow \infty$, and $\alpha$ is locally bounded; so $\alpha(u) /(1+u)^{\mu+2}$ $E \rightarrow \epsilon L(0, \infty)$. Also $\alpha(x \pm 0)$ exist for each positive $x$. Theorem 5 thus gives

$$
E \rightarrow \frac{1}{2}\{\alpha(x+0)+\alpha(x-0)\} \text { as } \eta \rightarrow+0 .
$$

If $\alpha(0) \neq 0$ we replace $\alpha(u)$ by $\alpha(u)-\alpha(0)$; then (6.6) becomes (6.5).

If $x=0$ the above application of Theorem 5 gives, instead of (6.6), that $E \rightarrow \frac{1}{2} \alpha(+0)$, and replacement of $\alpha(u)$ by $\alpha(u)-\alpha(0)$ then gives

$$
E \rightarrow \frac{1}{2}\{\alpha(+0)-\alpha(0)\}=\frac{1}{2}\{\alpha(+0)+\alpha(-0)\}-\alpha(0)
$$

if we agree to write $\alpha(-0)=\alpha(0)$.

COROLlaRY 6. If $\mathrm{re} p>-n$, where $n$ is a positive integer and $p$ is not zero or a negative integer, and if the other hypotheses of Theorem 6 hold, then for each positive $x$

$$
\begin{aligned}
& \frac{1}{2}\{\alpha(x+0)+\alpha(x-0)\}-\alpha(0) \\
& \quad=\lim _{\eta \rightarrow+0} \frac{(-1)^{n}}{2 \pi i(p)_{n}} \int_{-x}^{\lambda}(x+t)^{p+n-1}\left\{f^{(n)}(t-i \eta)-f^{(n)}(t+i \eta)\right\} d t,
\end{aligned}
$$

where $(p)_{n}$ has the meaning assigned in (4.7).

Proof. As for Corollary 4, using (5.12).

\section{Another inversion formula}

Comparing Theorems 4 and 6, particularly (5.1) and (6.5), we ask whether $\lambda=\infty$ is possible in the latter. The answer is given by Theorem 7 . This contains the most streamlined of our inversion formulae; but it also has the most restrictive hypothesis, in that it requires that $\alpha(t) /\left(1+t^{2}\right) \in L(0, \infty)$.

THEOREM 7. If re $p>0, \alpha$ is of bounded variation locally in $[0, \infty)$, $\alpha(t) /\left(1+t^{2}\right) \in L(0, \infty)$ and the integral

$$
f(s)=\int_{0}^{\rightarrow \infty} \frac{d \alpha(t)}{(s+t)^{p}}
$$

converges, then for each positive $x$ 


$$
\begin{aligned}
& \frac{1}{2}\{\alpha(x+0)+\alpha(x-0)\}-\alpha(0) \\
& \quad=\lim _{\eta \rightarrow+0} \frac{1}{2 \pi i} \int_{-x}^{\infty}(x+t)^{p-1}\{f(t-i \eta)-f(t+i \eta)\} d t .
\end{aligned}
$$

If $x=0$ this still holds with $\alpha(-0)$ replaced by $\alpha(0)$.

Proof. After Theorem 6 it is sufficient to suppose that $\alpha(0)=0$, to choose $\lambda>0$ and $x \geqq 0$, and to prove that

$$
J=\int_{\lambda}^{\infty}(x+t)^{p-1}\{f(t-i \eta)-f(t+i \eta)\} d t \rightarrow 0 \text { as } \eta \rightarrow+0 .
$$

Assuming the existence of this integral, which will follow from the inequalities below, Corollary 3 gives, as for $E_{1}$ in proving Theorem 4,

$$
J=\int_{\lambda}^{\infty}(x+t)^{p-1} d t \int_{0}^{\rightarrow \infty}\left(\frac{1}{(u+t-i \eta)^{p+1}}-\frac{1}{(u+t+i \eta)^{p+1}}\right) p \alpha(u) d u
$$

Supposing that $\eta>0$, and writing $\mu=$ re $p>0$, Lemma 3 gives

$$
\begin{aligned}
|J| & \leqq \int_{\lambda}^{\infty}(x+t)^{\mu-1} d t \int_{0}^{\infty} \frac{B \eta}{(u+t)^{\mu+2}}|p \alpha(u)| d u \\
& =|p| B \eta \int_{0}^{\infty}|\alpha(u)| d u \int_{\lambda}^{\infty} \frac{(x+t)^{\mu-1}}{(u+t)^{\mu+2}} d t .
\end{aligned}
$$

Now choosing $R>0$, and putting $t=u s$ when $u>R$,

$$
\left|\frac{J}{p B \eta}\right| \leqq \int_{0}^{R}|\alpha(u)| d u \int_{\lambda}^{\infty} \frac{(x+t)^{\mu-1}}{t^{\mu+2}} d t+\int_{R}^{\infty} \frac{|\alpha(u)|}{u^{2}} d u \int_{\lambda}^{\infty} \frac{(x+u s)^{\mu-1}}{u^{\mu-1}(1+s)^{\mu+2}} d s .
$$

The first of these double integrals is clearly finite, and the second is equal to

$$
\begin{aligned}
\int_{R}^{\infty} & \frac{|\alpha(u)|}{u^{2}} d u \int_{\lambda}^{\infty}\left(\frac{x}{u}+s\right)^{\mu-1} \frac{1}{(1+s)^{\mu+2}} d s \\
& \leqq \int_{R}^{\infty} \frac{|\alpha(u)|}{u^{2}} d u \int_{\lambda}^{\infty}\left\{\left(\frac{x}{R}+s\right)^{\mu-1}+s^{\mu-1}\right\} \frac{1}{(1+s)^{\mu+2}} d s<\infty
\end{aligned}
$$

here the first term in the braces supplies the necessary dominance if $\mu \geqq 1$, the second if $\mu \leqq 1$.

This shows that $J=O(\eta)$, and so completes the proof.

Remarks. 1. The expressions leading to (7.3) are like $K_{3}+K_{4}$ in the prof of Theorem 4, except that $(t+x)^{p-1}$ replaces $(t+x)^{p-1}-t^{p-1}$. This difference is the reason why the more restrictive hypothesis that $\alpha(t) /\left(1+t^{r}\right) \in L(0, \infty)$ with $r=2$ is required in Theorem 7, whereas $r=3$ is sufficient in Theorem 4. Another 
difference between the proofs of these two theorems is that change of order of integration is not needed in (7.2), whereas it is needed at (5.2) and (5.4).

2. Theorem 4 is in part a consequence of Theorem 7. For the case $x=0$ of Theorem 7 has the conclusion

$$
\frac{1}{2}\{\alpha(+0)-\alpha(0)\}=\lim _{n \rightarrow+0} \frac{1}{2 \pi i} \int_{0}^{\infty} t^{p-1}\{f(t-i \eta)-f(t+i \eta)\} d t
$$

and the lower terminal in this integral can be changed to $-x$, where $x>0$, if we introduce a Heaviside factor $H(t)$ into the integrand. Subtracting (7.4), so modified, from (7.1) we obtain (5.1).

3. The preceding paragraph is in fact a valid proof of the special case of Theorem 4 in which $\alpha(t) /\left(1+t^{2}\right) \in L(0, \infty)$. For the work of $\S 6$ and $\S 7$, although suggested by $\$ 5$, is logically independent of it (or could be so written). This would not prove the general case of Theorem 4 , but at least it shows a consistency between Theorems 4 and 7 which serves as a check on our work.

COROLlary 7. If re $p>-n$, where $n$ is a positive integer and $p$ is not zero or a negative integer, and if the other hypotheses of Theorem 7 hold, then (6.7) with $\lambda$ replaced by $\infty$ also holds.

\section{Relations with Sumner's work}

While our inversion formulae for (1.1) have little resemblance to the corresponding formula (1.3) of Sumner [2, page 181, Theorem $4 \mathrm{~b}]$, there is some resemblance between our (6.5) and his inversion formula for the transform $[2$, page $174,(4)]$

$$
g(s)=\int_{0}^{\rightarrow \infty} \frac{\phi(t)}{(s+t)^{p}} d t,
$$

even though they invert different transforms!

Sumner's inversion formula for $(8.1)$ is contained in [2, page 180, Theorem 4a] which is, restated in our notation:

THEOREM D. If $p>0, \phi$ is locally integrable in $[0, \infty)$, the improper Lebesgue integral (8.1) converges (for a certain s in the cut plane, and so for all), $x>0$ and the limits $\phi(x \pm 0)$ exist, then

$$
\frac{1}{2}\{\phi(x+0)+\phi(x-0)\}=\lim _{\eta \rightarrow+0} \frac{-1}{2 \pi i} \int_{c_{n x}}(x+z)^{p-1} g^{\prime}(z) d z,
$$

where $C_{\eta x}$ is a contour in the cut plane from $-x-i \eta$ to $-x+i \eta$.

Consider the prospect of deducing Theorem 6 , in the case of real $p$, from Theorem D. Suppose that the hypotheses of Theorem 6 hold and that also $\alpha(0)=0$. Corollary 3 gives that 


$$
f(s)=\int_{0}^{\rightarrow \infty} \frac{p \alpha(t)}{(s+t)^{p+1}} d t
$$

this improper $L$-integral converging. Comparing (8.1) with (8.3), Theorem D with $p$ replaced by $p+1$ gives, for all $x>0$,

$$
p \cdot \frac{1}{2}\{\alpha(x+0)+\alpha(x-0)\}=\lim _{\eta \rightarrow+0} \frac{-1}{2 \pi i} \int_{C(\eta, x, \lambda)}(x+z)^{p} f^{\prime}(z) d z,
$$

where $\lambda>0$ and $C(\eta, x, \lambda)$ is the three-segment polygonal contour with successive vertices $-x-i \eta, \lambda-i \eta, \lambda+i \eta,-x+i \eta$. Integrating by parts and then expressing the contour integral in terms of "real" integrals,

$$
\begin{aligned}
& \frac{1}{2}\{\alpha(x+0)+\alpha(x-0)\}=\lim _{\eta \rightarrow+0} \frac{1}{2 \pi i}\left\{\frac{(-i \eta)^{p}}{p} f(-x-i \eta)-\frac{(i \eta)^{p}}{p} f(-x+i \eta)\right. \\
& \left.\quad+\int_{-x}^{\lambda}(x+t-i \eta)^{p-1} f(t-i \eta) d t-\int_{-x}^{\lambda}(x+t+i \eta)^{p-1} f(t+i \eta) d t\right\} .
\end{aligned}
$$

We have omitted the integral along the segment from $\lambda-$ in to $\lambda+i \eta$; its integrand is bounded in a neighbourhood of $\lambda$, so the integral is $O(\eta)$ and contributes nothing to the limit as $\eta \rightarrow+0$.

To establish Theorem 6 from (8.4) it would be sufficient to show that

$$
(-i \eta)^{p} f(-x-i \eta)-(i \eta)^{p} f(-x+i \eta) \rightarrow 0
$$

and

$$
\begin{aligned}
\int_{-x}^{i} & {\left[\left\{(x+t-i \eta)^{p-1}-(x+t)^{p-1}\right\} f(t-i \eta)\right.} \\
& \left.-\left\{(x+t+i \eta)^{p-1}-(x+t)^{p-1}\right\} f(t+i \eta)\right] d t \rightarrow 0
\end{aligned}
$$

as $\eta \rightarrow+0$. Possibly (8.6) could be dealt with by methods like those of the foregoing proofs, although more lemmas would be needed. But (8.5) raises questions of the existence of $f(-x \pm i 0)$, or of the order of magnitude of $f(-x \pm i \eta)$ for small $\eta$, and these seem to be elusive.

It appears then that our inversion formulae are not very close to Sumner's. This view is further supported by the observation that our Lemma 8 holds for all $p$, whereas Sumner's Lemma $4 c$, a result of a similar character, fails for $p=0$ and $p=-1$ (at least).

Theorem D raises another question. Do our methods also lead to inversion formulae for the transform (8.1)? Our methods have indeed depended on (8.3) which resembles (8.1); but (8.1) differs significantly when $\phi$ is not locally of bounded variation. Theorem 6 with

$$
\alpha(t)=\int_{0}^{t} \phi(u) d u
$$


gives the right side of (6.5) as an expression for $\alpha(x)$, but this only gives the rather unattractive formula

$$
\phi(x)=\frac{d}{d x} \lim _{\eta \rightarrow+0} \frac{1}{2 \pi i} \int_{-}^{\lambda}(x+t)^{p-1}\{f(t-i \eta)-f(t+i \eta)\} d t
$$

for almost all positive $x$, unless the differentiation and limit operations can be commuted. A different approach is needed.

Before doing that we digress, in the interests of generality, to define Lebesgue left and right limits $\phi(x \pm 0)$ of a locally integrable function $\phi$, extending the familiar concept of left and right limits. The extended concept was implicitly used by Titchmarsh [6, page 28, Theorem 13], but it appears to have since been neglected.

We denote by $\phi(x+0)$ a number $l_{+}$, if it exists, such that

$$
\frac{1}{h} \int_{0}^{h}\left|\phi(x+u)-l_{+}\right| d u \rightarrow 0 \text { as } h \rightarrow+0 .
$$

Such a number $l_{+}$, namely $\phi(x)$, exists for all Lebesgue points $x$ of $\phi$, and possibly at other points. There cannot be two such numbers $l_{1}$ and $l_{2}$ for the same $x$, because

$$
\begin{aligned}
\left|l_{2}-l_{1}\right| & =\frac{1}{h} \int_{0}^{h}\left|l_{2}-l_{1}\right| d u \\
& \leqq \frac{1}{h} \int_{0}^{h}\left|l_{2}-\phi(x+u)\right| d u+\frac{1}{h} \int_{0}^{h}\left|\phi(x+u)-l_{1}\right| d u \rightarrow 0
\end{aligned}
$$

as $h \rightarrow+0$. Thus $\phi(x+0)$ is unique if it exists; and it exists almost everywhere. And it exists and agrees with the usual $\phi(x+0)$ wherever this exists.

Similarly $\phi(x-0)$ denotes a number $l_{-}$, if it exists, such that

$$
\frac{1}{h} \int_{0}^{h}\left|\phi(x+u)-l_{-}\right| d u \rightarrow 0 \text { as } h \rightarrow-0 .
$$

It is unique if it exists; it exists almost everywhere; and it is consistent with the usual left limit $\phi(x-0)$. The Lebesgue points of $\phi$ are the points $x$ at which $\phi(x-0)=\phi(x)=\phi(x+0)$.

Theorem 5 and Lemma 8 hold with this extended meaning of $\phi(x \pm 0)$, because Cauchy's singular integral does.

THEOREM 8. If re $p>1, \phi$ is locally integrable in $[0, \infty)$, the improper L-integral

$$
g(s)=\int_{0}^{\rightarrow \infty} \frac{\phi(t)}{(s+t)^{p}} d t
$$


converges, and $\lambda>0$; then, for each positive $x$ for which the Lebesgue limits $\phi(x \pm 0)$ exist, and in particular for almost all positive $x$,

(8.8) $\frac{1}{2}\left\{\phi(x+0)+\phi(x-0\}=\lim _{\eta \rightarrow+0} \frac{p-1}{2 \pi i} \int_{-x}^{\lambda}(x+t)^{p-2}\{g(t-i \eta)-g(t+i \eta)\} d t\right.$.

Proof. Let $\alpha$ be an indefinite integral of $\phi$. For some $s$ in the cut plane

$$
\int_{0}^{\rightarrow \infty} \frac{d \alpha(t)}{(s+t)^{p}}=\lim _{T \rightarrow \infty} \int_{0}^{T} \frac{\phi(t)}{(s+t)^{p}} d t=\int_{0}^{\rightarrow \infty} \frac{\phi(t)}{(s+t)^{p}} d t
$$

converges; so by Theorem 1 it converges for all $s$ in the cut plane. By Corollary 2 $g$ is continuous in the cut plane; thus the integral $I$ in (8.8) exists, since also re $p>1$. Further Theorem 3 shows that

$$
\alpha(t)=o\left(t^{p}\right) \text { as } t \rightarrow \infty ;
$$

so that, writing $\mu=$ re $p$, there is $R>x$ such that

$$
|\alpha(t)| \leqq \frac{1}{2} t^{\mu} \text { for all } t \geqq R \text {. }
$$

The integral $I$ in (8.8) is, by (8.1),

$$
I=\int_{-x}^{\lambda}(x+t)^{p-2} d t \int_{0}^{\rightarrow \infty}\left(\frac{1}{(u+t-i \eta)^{p}}-\frac{1}{(u+t+i \eta)^{p}}\right) \phi(u) d u .
$$

Let $I_{1}$ and $I_{2}$ be the same double integrals except that the inner terminals are 0 and $R$ for $I_{1}$, and $R$ and $\rightarrow \infty$ for $I_{2}$. Existence of $I_{2}$ will be evident in a moment, hence also that of $I_{1}$; and $I=I_{1}+I_{2}$.

Integrating by parts the inner integral in $I_{2}$, and using Lemma 3 and (8.10),

$$
\begin{aligned}
& I_{2}=\int_{-x}^{\lambda}(x+t)^{p-2} d t \int_{R}^{\rightarrow \infty} p\left(\frac{1}{(u+t-i \eta)^{p+1}}-\frac{1}{(u+t+i \eta)^{p+1}}\right)\{\alpha(u)-\alpha(R)\} d u \\
&\left|I_{2}\right| \leqq \int_{-x}^{\lambda}(x+t)^{\mu-2} d t \int_{R}^{\rightarrow \infty}|p| \frac{B \eta}{|u+t|^{\mu+2}} \frac{u^{\mu}+R^{\mu}}{2} d u \\
& \leqq \int_{-x}^{\lambda}(x+t)^{\mu-2} d t \int_{R}^{\infty} \frac{|p| B \eta}{(u+t)^{\mu+2}} u^{\mu} d u \\
& \leqq|p| B \eta \int_{-x}^{\lambda}(x+t)^{\mu-2} d t \int_{R}^{\infty} \frac{u^{\mu}}{(u-x)^{\mu+2}} d u=O(\eta) \quad \text { as } \eta \rightarrow+0
\end{aligned}
$$

Changing the order of integration in $I_{1}$, with justification later,

$$
I_{1}=\int_{0}^{R} \phi(u) d u \int_{-x}^{\lambda}\left(\frac{(x+t)^{p-2}}{(u+t-i \eta)^{p}}-\frac{(x+t)^{p-2}}{(u+t+i \eta)^{p}}\right) d t
$$




$$
(p-1) I_{1}=\int_{0}^{R}\left(\frac{1}{u-x-i \eta} \frac{(x+\lambda)^{p-1}}{(u+\lambda-i \eta)^{p-1}}-\frac{1}{u-x+i \eta} \frac{(x+\lambda)^{p-1}}{(u+\lambda+i \eta)^{p-1}}\right) \phi(u) d u
$$

where we have used Lemma 5 and the datum that re $p>1$. Now Lemma 8 gives

$$
\frac{p-1}{2 \pi i} I_{1} \rightarrow \frac{1}{2}\{\phi(x+0)+\phi(x-0)\} \text { as } \eta \rightarrow+0 .
$$

This, with $I_{2} \rightarrow 0$ as shown above, establishes (8.8) subject to justifying (8.11). The justification is provided by showing that (8.11) is absolutely convergent. For this we have, using Lemma 1 ,

$$
\begin{gathered}
\left|(u+t \pm i \eta)^{-p}\right| \leqq|u+t \pm i \eta|^{-\mu} e^{\pi|v|} \leqq \eta^{-\mu} e^{\pi|v|} \\
\int_{0}^{R}|\phi(u)| d u \int_{-x}^{\lambda}\left|\frac{1}{(u+t-i \eta)^{p}}-\frac{1}{(u+t+i \eta)^{p}}\right|\left|(x+t)^{p-2}\right| d t \\
\leqq 2 \eta^{-\mu} e^{\pi|v|} \int_{0}^{R}|\phi(u)| d u \int_{-x}^{\lambda}(x+t)^{\mu-2} d t<\infty
\end{gathered}
$$

This completes the proof. A shorter proof might have been expected by not dissecting $I$ into $I_{1}$ and $I_{2}$, but applying to $I$ the treatment given to $I_{1}$. Then $I$ would be given by (8.11) and (8.12) with $R$ replaced by $\infty$. But Theorem 5 woudl not then be applicable to (8.12), for lack of the hypothesis that

$$
\phi(u) /(1+u)^{p+2} \in L(0, \infty) .
$$

COROLlaRY 8. If re $p>1-n$, where $n$ is a positive integer and $p-1$ is not zero or a negative integer, and if the other hypotheses of Theorem 8 hold, then for each positive $x$ for which the Lebesgue limits $\phi(x \pm 0)$ exist

$$
\begin{aligned}
& \frac{1}{2}\{\phi(x+0)+\phi(x-0)\} \\
& \quad=\lim _{n \rightarrow+0} \frac{(-1)^{n}}{2 \pi i(p)_{n-1}} \int_{-x}^{\lambda}(x+t)^{p+n-2}\left\{g^{(n)}(t-i \eta)-g^{(n)}(t+i \eta)\right\} d t
\end{aligned}
$$

where $(p)_{n-1}$ has the meaning assigned in (4.7).

Proof. By Corollary 2, (8.1) and (8.9),

$$
\frac{g^{(n)}(s)}{(-1)^{n}(p)_{n}}=\int_{0}^{\rightarrow \infty} \frac{\phi(t)}{(s+t)^{p+n}} d t
$$

division by $(p)_{n}$ being valid since $(p)_{n} \neq 0$. Then (8.14) follows from inverting this by Theorem 8 .

Remark. Our nearest approach to Theorem D is the case $n=1$ of Corollary 8 , which may be restated as follows: 
If re $p>0$ and $p \neq 1, \phi$ is locally integrable in $[0, \infty)$, the improper L-integral

$$
g(s)=\int_{0}^{+\infty} \frac{\phi(t)}{(s+t)^{p}} d t
$$

converges, and $\lambda>0$; then, for each positive $x$ for which the Lebesgue limits $\phi(x \pm 0)$ exist, and so for almost all positive $x$,

$$
\begin{aligned}
& \frac{1}{2}\{\phi(x+0)+\phi(x-0)\} \\
& \quad=\lim _{\eta \rightarrow+0} \frac{-1}{2 \pi i} \int_{-x}^{i}(x+t)^{p-1}\left\{g^{\prime}(t-i \eta)-g^{\prime}(t+i \eta)\right\} d t .
\end{aligned}
$$

This inversion formula would become Sumner's, that is (8.2), if we could replace the integrand by

$$
(x+t-i \eta)^{p-1} g^{\prime}(t-i \eta)-(x+t+i \eta)^{p-1} g^{\prime}(t+i \eta) ;
$$

for by adding an $O(\eta)$ term the integral would then become a contour integral along $C(\eta, x, \lambda)$ (described in connection with (8.3) above), and this would be deformable into $C_{\eta x}$. We would then have Theorem $\mathrm{D}$ under wider conditions: $p$ complex, and more values of $x$.

In the missing case $p=1$ the above theorem still holds, for (8.15) reduces to Widder's complex inversion formula [1, page 340, Theorem $7 \mathrm{~b}]$.

Theorem 8 leads to Theorem 9 (below) in the same way as Theorem 6 led to Theorem 7 .

THEOREM 9. If re $p>1, \phi(t) /\left(1+t^{2}\right) \in L(0, \infty)$ and the improper L-integral

$$
g(s)=\int_{0}^{\rightarrow \infty} \frac{\phi(t)}{(s+t)^{p}} d t
$$

converges, then for each positive $x$ for which the Lebesgue limits $\phi(x \pm 0)$ exist, $\frac{1}{2}\{\phi(x+0)+\phi(x-0)\}=\lim _{\eta \rightarrow+0} \frac{p-1}{2 \pi i} \int_{-x}^{\infty}(x+t)^{p-2}\{g(t-i \eta)-g(t+i \eta)\} d t$.

Proof. After Theorem 8 it is sufficient to choose $\lambda>0$ and $x>0$ and to prove that $J \rightarrow 0$ as $\eta \rightarrow+0$, where

$$
J=\int_{\lambda}^{\infty}(x+t)^{p-2} d t \int_{0}^{\rightarrow \infty}\left(\frac{1}{(u+t-i \eta)^{p}}-\frac{1}{(u+t+i \eta)^{p}}\right) \phi(u) d u .
$$

This expression becomes (7.2) if we replace $p$ by $p+1$ and $\phi$ by $p \alpha$. In the proof of Theorem 7 from (7.2) onwards, the only properties of $p$ and $\alpha$ that are used are that re $p>0$ and that $\alpha(u) /\left(1+u^{2}\right) \in L(0, \infty)$. So that proof completes this proof of Theorem 9. 
COROLlaRY 9. If re $p>1-n$, where $n$ is a positive integer and $p-1$ is not zero or a negative integer, and if the other hypotheses of Theorem 9 hold, then (8.14), with $\lambda$ replaced by $\infty$, holds for each positive $x$ for which the Lebesgue limits $\phi(x \pm 0)$ exist.

\section{Extension to generalized bounded variation}

The kind of generalized total variation we now consider was first used by Wiener [7]. It was developed by Young and Love [8,9 and 10] in connection with Stieltjes integrals, and led to conditions of integrability symmetrical between the two functions involved, as in Lemma 12. Their work was generalized still further by Musielak and Orlicz [11].

We aim to show that, throughout the present paper, the adjective "of bounded variation" can be replaced by "of bounded $q$ th power variation, with $q \geqq 1$ ". The changes needed are almost all in the proof of Theorem 1; we detail them after stating, and where necessary proving, the requisite properties of this generalized total variation.

One effect of this generalization is that even the proper Stieltjes integrals on $(0, R)$ cease to be absolutely convergent. The improper Stieltjes integrals on $(0, \rightarrow \infty)$ have of course been permitted to be conditionally convergent throughout, but the generalization adds a new dimension to this conditional convergence.

For conformity with the notation of the literature, we shall in this section use $p$ to mean a real number quite independent of the complex parameter $p$ in (1.1). Similarly $f$ and $g$ will be freed from their previous meanings.

DEFINITION. For $p \geqq 1$, and complex-valued functions $f$ on a compact interval $[a, b]$, define the pth power variation of $f$ on $[a, b]$ as

$$
V_{p}(f)=V_{p}(f ; a, b)=\sup \left(\sum_{i=1}^{n}\left|f\left(x_{i}\right)-f\left(x_{i-1}\right)\right|^{p}\right)^{1 / p},
$$

taking the upper bound for all subdivisions $a=x_{0}<x_{1}<\cdots<x_{n}=b$. (As in [8, page 258] or [9, page 243].)

Also define $W_{p}=W_{p}(a, b)=\left\{f: V_{p}(f ; a, b)<\infty\right\}$.

In particular, $V_{1}(f)$ is the ordinary total variation of $f$, and $W_{1}$ is the ordinary class of functions of bounded variation. For $p>1, W_{p} \supset W_{1}$; more generally $W_{p}$ expands as $p$ increases, because $V_{p}(f)$ decreases [8, page 258]. Thus the classes of functions we discuss are wider than the class of functions of bounded variation.

All functions $f \in W_{p}$ are bounded, and their ordinary left and right limits $f(x-0)$ and $f(x+0)$ exist for all $x$ concerned. This is proved in just the same way as for $p=1$. 
Lemma 9. If $p \geqq 1$ and $a<c<b$, then

$$
V_{p}(f ; a, c)^{p}+V_{p}(f ; c, b)^{p} \leqq V_{p}(f ; a, b)^{p} .
$$

Similarly for subdivision of $[a, b]$ into any finite family of subintervals.

Proof. Almost as for $p=1$.

LEMMA 10. If $p \geqq 1, f \in W_{p}(a, b)$ and $f$ is continuous, then $V_{p}(f ; a, x)$ is increasing and continuous in $a \leqq x \leqq b$.

ProOF. Almost as for $p=1$. For part of such a proof see [12, page 249].

Lemma 11. If $p \geqq 1, f \in W_{p}$ and $g \in W_{p}$, then $f g \in W_{p}$ and

$$
V_{p}(f g) \leqq V_{p}(f) B(g)+B(f) V_{p}(g),
$$

where all variations are on $[a, b]$ and $B(f)=B(f ; a, b)=\sup \{|f(x)|: a \leqq x \leqq b\}$.

Proof. Suppose $a=x_{0}<x_{1}<\cdots<x_{n}=b$. Using Minkowski's inequality,

$$
\begin{aligned}
& \left(\sum_{i=1}^{n}\left|f\left(x_{i}\right) g\left(x_{i}\right)-f\left(x_{i-1}\right) g\left(x_{i-1}\right)\right|^{p}\right)^{1 / p} \\
& \leqq\left(\sum_{i=1}^{n}\left\{\left|f\left(x_{i}\right)-f\left(x_{i-1}\right)\right|\left|g\left(x_{i}\right)\right|+\left|f\left(x_{i-1}\right)\right|\left|g\left(x_{i}\right)-g\left(x_{i-1}\right)\right|\right\}^{p}\right)^{1 / p} \\
& \leqq\left(\sum_{i=1}^{n}\left|f\left(x_{i}\right)-f\left(x_{i-1}\right)\right|^{p}\left|g\left(x_{i}\right)\right|^{p}\right)^{1 / p}+\left(\sum_{i=1}^{n}\left|f\left(x_{i-1}\right)\right|^{p}\left|g\left(x_{i}\right)-g\left(x_{i-1}\right)\right|^{p}\right)^{1 / p} \\
& \leqq\left(\sum_{i=1}^{n}\left|f\left(x_{i}\right)-f\left(x_{i-1}\right)\right|^{p}\right)^{1 / p} B(g)+B(f)\left(\sum_{i=1}^{n}\left|g\left(x_{i}\right)-g\left(x_{i-1}\right)\right|^{p}\right)^{1 / p}
\end{aligned}
$$$$
\leqq V_{p}(f) B(g)+B(f) V_{p}(g) \quad \text { for all subdivisions, as required. }
$$

Next we state Young and Love's symmetrical existence theorem for R!̣emann-Stieltjes integrals.

LEMMA 12. If $p \geqq 1, q \geqq 1, p^{-1}+q^{-1}>1, f \in W_{p}(a, b)$ and $g \in W_{q}(a, b)$, $f$ and $g$ have no common discontinuity in $[a, b]$, then the Riemann-Stieltjes integral of $f$ with respect to $g$ on $[a, b]$ exists; and, if $a \leqq c \leqq b$,

$$
\left|\int_{a}^{b}\{f(x)-f(c)\} d g(x)\right| \leqq K V_{p}(f ; a, b) V_{q}(g ; a, b)
$$

where $K$ depends only on $p$ and $q$. (A possible value of $K$ is $1+\zeta\left(p^{-1}+q^{-1}\right)$, where $\zeta$ is Riemann's Zeta function.)

Proof in [8] extends over pages 254 to 257 and 263 to 265 . For the statement, see [8, page 265 and 266$]$ or [9, page 246$]$. 
THEOREM 10. If $\alpha, f$ and $g$ are functions on $[a, b], f$ is continuous, $g$ and $\alpha$ have no common discontinuity, $f$ and $g$ are in $W_{p}$, $\alpha$ is in $W_{q}, p \geqq 1, q>1$, $p^{-1}+q^{-1}>1$, and

then $\beta$ is in $W_{q}$ and

$$
\beta(x)=\int_{a}^{x} g(t) d \alpha(t) \quad \text { for } a \leqq x \leqq b,
$$

$$
\int_{a}^{b} f(x) d \beta(x)=\int_{a}^{b} f(x) g(x) d \alpha(x) .
$$

Proof. (i) $\beta(x)$ exists for $a \leqq x \leqq b$, by Lemma 12. Supposing that $a \leqq x<x^{\prime} \leqq b$,

$$
\begin{aligned}
\left|\beta\left(x^{\prime}\right)-\beta(x)\right|=\mid \int_{x}^{x^{\prime}} & g(t) d \alpha(t)|\leqq| \int_{x}^{x^{\prime}}\{g(t)-g(x)\} d \alpha(t)|+| \int_{x}^{x^{\prime}} g(x) d \alpha(t) \mid \\
& \leqq K V_{p}\left(g ; x, x^{\prime}\right) V_{q}\left(\alpha ; x, x^{\prime}\right)+|g(x)|\left|\alpha\left(x^{\prime}\right)-\alpha(x)\right| \\
& \leqq K V_{p}(g ; a, b) V_{q}\left(\alpha ; x, x^{\prime}\right)+M V_{q}\left(\alpha ; x, x^{\prime}\right) \\
& =L V_{q}\left(\alpha ; x, x^{\prime}\right),
\end{aligned}
$$

where Lemma 12 has been used, $K$ is the constant occurring in it, $M=B(g)$ is as defined in Lemma 11, and $L$ is independent of $x$ and $x^{\prime}$. Now supposing that $a=x_{0}<x_{1}<\cdots<x_{n}=b$,

$$
\left(\sum_{i=1}^{n}\left|\beta\left(x_{i}\right)-\beta\left(x_{i-1}\right)\right|^{q}\right)^{1 / q} \leqq L\left(\sum_{i=1}^{n} V_{q}\left(\alpha ; x_{i-1}, x_{i}\right)^{q}\right)^{1 / q} \leqq L V_{q}(\alpha ; a, b)
$$

using Lemma 9. Thus $V_{q}(\beta ; a, b) \leqq L V_{q}(\alpha ; a, b)$ and $\beta \in W_{q}$.

(ii) By Lemma 11, $f g \in W_{p}$. The discontinuities of $f g$ are those of $g$ at most, so they are not discontinuities of $\alpha$. So Lemma 12 gives the existence of the integral on the right in (9.1); in fact it gives the existence of both integrals in (9.1), though we shall prove independently (and incidentally) that the one on the left exists. Let

and

$$
a=x_{0} \leqq \xi_{1} \leqq x_{1} \leqq \xi_{2} \leqq x_{2} \leqq \cdots \leqq x_{n-1} \leqq \xi_{n} \leqq x_{n}=b
$$

$$
\begin{aligned}
D & =\int_{a}^{b} f(x) g(x) d \alpha(x)-\sum_{i=1}^{n} f\left(\xi_{i}\right)\left\{\beta\left(x_{i}\right)-\beta\left(x_{i-1}\right)\right\} \\
& =\sum_{i=1}^{n}\left(\int_{x_{i}-1}^{x_{i}} f(x) g(x) d \alpha(x)-f\left(\xi_{i}\right) \int_{x_{i}-1}^{x_{i}} g(x) d \alpha(x)\right) \\
& =\sum_{i=1}^{n} \int_{x_{i}-1}^{x_{i}}\left\{f(x)-f\left(\xi_{i}\right)\right\} g(x) d \alpha(x) \\
& =\sum_{i=1}^{n} \int_{x_{i-1}}^{x_{i}}\left\{f_{i}(x) g(x)-f_{i}\left(\xi_{i}\right) g\left(\xi_{i}\right)\right\} d \alpha(x),
\end{aligned}
$$


where

$$
f_{i}(x)=f(x)-f\left(\xi_{i}\right) \quad \text { for } x_{i-1} \leqq x \leqq x_{i} .
$$

By Lemma $11, f_{i} g \in W_{p}\left(x_{i-1}, x_{i}\right)$; and so by Lemma 12 with $c=\xi_{i}$,

$$
|D| \leqq \sum_{i=1}^{n} K V_{p}\left(f_{i} g ; x_{i-1}, x_{i}\right) V_{q}\left(\alpha ; x_{i-1}, x_{i}\right) \text {. }
$$

By Lemma $10, V_{p}(f ; a, x)^{p}$ is increasing and continuous in $a \leqq x \leqq b$. So it is uniformly continuous and there is $\delta=\delta(\varepsilon)>0$ such that

$$
0 \leqq V_{p}\left(f ; a, x^{\prime}\right)^{p}-V_{p}(f ; a, x)^{p}<\varepsilon^{p} \text { if } x^{\prime}-x<\delta \text { and } a \leqq x \leqq x^{\prime} \leqq b .
$$

But by Lemma 9 ,

$$
V_{p}\left(f ; x, x^{\prime}\right)^{p} \leqq V_{p}\left(f ; a, x^{\prime}\right)^{p}-V_{p}(f ; a, x)^{p},
$$

and so

$$
\left|f\left(x^{\prime}\right)-f(x)\right|^{p} \leqq V_{p}\left(f ; x, x^{\prime}\right)^{p}<\varepsilon^{p} \text { if } x^{\prime}-x<\delta \text { and } a \leqq x \leqq x^{\prime} \leqq b .
$$

By Lemma 11 and (9.4), all bounds and variations being on $\left[x_{i-1}, x_{i}\right]$,

$$
V_{p}\left(f_{i} g\right) \leqq V_{p}\left(f_{i}\right) B(g)+B\left(f_{i}\right) V_{p}(g), \leqq M V_{p}(f)+\varepsilon V_{p}(g)
$$

so long as $x_{i}-x_{i-1}<\delta$.

Define $r$ by $r^{-1}+q^{-1}=1$, so that $\infty>r>1$; indeed, $r>p \geqq 1$. Suppose that $\max \left(x_{i}-x_{i-1}\right)<\delta$. By (9.3) and (9.5), and then by Hölder's inequality,

$$
\begin{aligned}
|D| \leqq & K \sum_{i=1}^{n}\left\{M V_{p}\left(f ; x_{i-1}, x_{i}\right)+\varepsilon V_{p}\left(g ; x_{i-1}, x_{i}\right)\right\} V_{q}\left(\alpha ; x_{i-1}, x_{i}\right) \\
\leqq & M K\left(\sum_{i=1}^{n} V_{p}\left(f ; x_{i-1}, x_{i}\right)^{r}\right)^{1 / r}\left(\sum_{i=1}^{n} V_{q}\left(\alpha ; x_{i-1}, x_{i}\right)^{q}\right)^{1 / q} \\
& +\varepsilon K\left(\sum_{i=1}^{n} V_{p}\left(g ; x_{i-1}, x_{i}\right)^{r}\right)^{1 / r}\left(\sum_{i=1}^{n} V_{q}\left(\alpha ; x_{i-1}, x_{i}\right)^{q}\right)^{1 / q} \\
\leqq & M K\left(\sum_{i=1}^{n} \varepsilon^{r-p} V_{p}\left(f ; x_{i-1}, x_{i}\right)^{p}\right)^{1 / r} V_{q}(\alpha ; a, b) \\
& +\varepsilon K\left(\sum_{i=1}^{n} V_{p}(g ; a, b)^{r-p} V_{p}\left(g ; x_{i-1}, x_{i}\right)^{p}\right)^{1 / r} V_{q}(\alpha ; a, b) .
\end{aligned}
$$

Here we have used the inequalities $V_{p}\left(f ; x_{i-1}, x_{i}\right)<\varepsilon$, derived from (9.4), and $V_{p}\left(g ; x_{i-1}, x_{i}\right) \leqq V_{p}(g ; a, b)$; the positiveness of $r-p$; and Lemma 9 to deal with the $q$ th power variations. Now using Lemma 9 again, this time for the $p$ th power variations,

$$
|D| \leqq\left\{M \varepsilon^{1-(p / r)} V_{p}(f ; a, b)^{p / r}+\varepsilon V_{p}(g ; a, b)^{1-(p / r)+(p / r)}\right\} K V_{q}(\alpha ; a, b) .
$$


Since $1-(p / r)>0$, this shows that $D \rightarrow 0$ as $\max \left(x_{i}-x_{i-1}\right) \rightarrow 0$. Considering (9.2), this proves that the integral on the left of (9.1) exists and that (9.1) holds.

The changes in $\$ 4$ entailed by supposing that $\alpha$ is of bounded $q$ th power variation locally in $[0, \infty)$, with $q>1$ instead of $q=1$, are as follows.

1. Equation (4.3) holds, and the integrals on both sides of it exist, by Theorem 10 with

$$
p=1, \quad f(t)=\frac{\left(s_{0}+t\right)^{p}}{(s+t)^{p}}, \quad g(t)=\frac{1}{\left(s_{0}+t\right)^{p}}
$$

(the complex parameter $p$ in $f$ and $g$ has no connection with the real $p$ in Theorem 10). Theorem 10 applies because $f$ and $g$ are continuous in $[0, R]$, indeed continuously differentiable and so in $W_{1}(0, R)$; and $p^{-1}+q^{-1}=1+q^{-1}>1$. This looks after the first paragraph of the proof of Theorem 1; and also the last sentence before Theorem 1, although the latter could also be established directly from Lemma 12.

2. That $\beta$ is of bounded $q$ th power variation locally in $[0, \infty)$ also follows from Theorem 10. This looks after the second paragraph of the proof of Theorem 1 , and also the last line of the statement.

3. There is a minor difference from the normal proof of Corollary 2, namely in showing that

$$
\int_{0}^{R} \frac{d \alpha(t)}{(s+t)^{p}}
$$

is a regular function of $s$ in the cut plane, for each $R>0$. This can be deduced from standard theory after integration by parts.

4. Equation (4.8) holds, like equation (4.3), by Theorem 10.

After $\S 4$ everything involving $\alpha$ is done by way of Corollary 3 , and all that is required of $\alpha$ is local integrability in $[0, \infty)$, apart from any other conditions imposed from time to time. Since $\alpha$ is assumed to be in $W_{q}(0, R)$ for each $R>0$, its discontinuities are simple, hence countable, hence they form a set of measure zero. Further $\alpha$ is bounded on each $(0, R)$. So it is $R$-integrable on each $(0, R)$, and hence locally $L$-integrable as required.

We thus conclude that all our results hold if for "bounded variation" we read "bounded $q$ th power variation, for any $q \geqq 1$ ".

\section{Acknowledgment}

One of us (E.R.L.) acknowledges the hospitality of Professor Cassels and the facilities of the Department of Pure Mathematics in Cambridge during the final stages of this work. 


\section{References}

[1] D. V. Widder, The Laplace Transform (Princeton 1946).

[2] D. B. Sumner, 'An inversion formula for the Generalized Stieltjes Transform', Bull. American Math. Soc. 55 (1949), 174-183.

[3] I. I. Hirschman and D. V. Widder, The Convolution Transform (Princeton 1955).

[4] J. N. Pandey and A. H. Zemanian, 'Complex inversion for the Generalized Convolution Transformation', Pacific Journ. of Math. 25 (1968), 147-157.

[5] A. Erdélyi and others, Tables of Integral Transforms, vol. 2, Bateman Manuscript Project (McGraw-Hill 1954).

[6] E. C. Titchmarsh, Theory of Fourier Integrals (Oxford 1937).

[7] N. Wiener, 'The Quadratic Variation of a function and its Fourier coefficients', J. Massachusetts Inst. of Tech. 3 (1924), 73-94.

[8] L. C. Young, 'An inequality of the Hölder type, connected with Stieltjes integration', Acta Math. 67 (1936), 251-282.

[9] E. R. Love et L. C. Young, 'Sur une classe de fonctionnelles linéaires', Fund. Math. 28 (1937), 243-257.

[10] E. R. Love and L. C. Young, 'On fractional integration by parts', Proc. London Math. Soc. (2) 44 (1938), 1-35.

[11] J. Musielak and W. Orlicz, 'On generalized variations (I)'. Studia Math. 18 (1959), 11-41.

[12] E. R. Love, 'Fractional derivatives of imaginary order', J. London Math. Soc. (2) 3 (1971), 241-259.

Department of Mathematics

State College of Victoria at Melbourne

Australia

and

Department of Mathematics

University of Melbourne

Porkville, 3052

Australia 